PAPER

\section{Hybrid nanomembrane-based capacitors for the determination of the dielectric constant of semiconducting molecular ensembles}

To cite this article: Paula A Petrini et al 2018 Nanotechnology 29265201

View the article online for updates and enhancements.
Related content

- Origin and role of gap states in organic
semiconductor studied by UPS: as the
nature of organic molecular crystals
Jin-Peng Yang, Fabio Bussolotti, Satoshi
Kera et al.
- The effect of gate dielectric modification
and film deposition temperature on the
field effect mobility of copper (II)
phthalocyanine thin-film transistors
Sumona Sinha, C-H Wang, M Mukherjee
et al.
- Flexible diodes for radio frequency (RF)
electronics: a materials perspective
James Semple, Dimitra G Georgiadou,
Gwenhivir Wyatt-Moon et al.

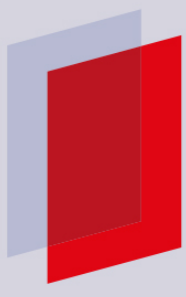

\section{IOP ebooks}

Bringing you innovative digital publishing with leading voices

to create your essential collection of books in STEM research.

Start exploring the collection - download the first chapter of every title for free. 


\title{
Hybrid nanomembrane-based capacitors for the determination of the dielectric constant of semiconducting molecular ensembles
}

\author{
Paula A Petrini ${ }^{1,2}$, Ricardo M L Silva ${ }^{1,2}$, Rafael F de Oliveira ${ }^{1}{ }^{(1)}$, \\ Leandro Merces $^{1,3}$ (1) and Carlos C Bof Bufon ${ }^{1,2,3}$ \\ ${ }^{1}$ Brazilian Nanotechnology National Laboratory (LNNano), Brazilian Center for Research in Energy and \\ Materials (CNPEM), 13083-970 Campinas, São Paulo, Brazil \\ ${ }^{2}$ Postgraduate Program in Materials Science and Technology (POSMAT), São Paulo State University \\ (UNESP), 17033-360 Bauru, São Paulo, Brazil \\ ${ }^{3}$ Institute of Physics 'Gleb Wataghin' (IFGW), University of Campinas (UNICAMP), 13083-859 \\ Campinas, São Paulo, Brazil \\ E-mail: cesar.bof@lnnano.cnpem.br
}

Received 1 February 2018, revised 26 March 2018

Accepted for publication 6 April 2018

Published 2 May 2018

\begin{abstract}
Considerable advances in the field of molecular electronics have been achieved over the recent years. One persistent challenge, however, is the exploitation of the electronic properties of molecules fully integrated into devices. Typically, the molecular electronic properties are investigated using sophisticated techniques incompatible with a practical device technology, such as the scanning tunneling microscopy. The incorporation of molecular materials in devices is not a trivial task as the typical dimensions of electrical contacts are much larger than the molecular ones. To tackle this issue, we report on hybrid capacitors using mechanicallycompliant nanomembranes to encapsulate ultrathin molecular ensembles for the investigation of molecular dielectric properties. As the prototype material, copper (II) phthalocyanine (CuPc) has been chosen as information on its dielectric constant $\left(k_{\mathrm{CuPc}}\right)$ at the molecular scale is missing. Here, hybrid nanomembrane-based capacitors containing metallic nanomembranes, insulating $\mathrm{Al}_{2} \mathrm{O}_{3}$ layers, and the CuPc molecular ensembles have been fabricated and evaluated. The $\mathrm{Al}_{2} \mathrm{O}_{3}$ is used to prevent short circuits through the capacitor plates as the molecular layer is considerably thin $(<30 \mathrm{~nm})$. From the electrical measurements of devices with molecular layers of different thicknesses, the CuPc dielectric constant has been reliably determined

$\left(k_{\mathrm{CuPc}}=4.5 \pm 0.5\right)$. These values suggest a mild contribution of the molecular orientation on the $\mathrm{CuPc}$ dielectric properties. The reported nanomembrane-based capacitor is a viable strategy for the dielectric characterization of ultrathin molecular ensembles integrated into a practical, real device technology.
\end{abstract}

Supplementary material for this article is available online

Keywords: molecular electronics, nanomembranes, dielectric properties, organic semiconductors, hybrid capacitors

(Some figures may appear in colour only in the online journal)

\section{Introduction}

Controlling the properties of organic devices at the molecular level has expanded electronics toward new developments
$[1,2]$. In the so-called molecular electronics, individual molecules and molecular ensembles are the building blocks of switches [3], transistors [4], photovoltaics [5], and other applications aimed to present functionalities unachievable 
with inorganic semiconductors [6]. The advancements in this field are strongly related to the intrinsic characteristics of molecules, for example, their inherent reduced size and flexibility [2]. Regarding electronic properties, the electronic charge is highly localized, with distribution dependent on the molecular configuration, which can lead to unique quantum effects [7]. Another appeal of molecular materials is the possibility to tailor properties through chemical synthesis, which is considered advantageous for customized applications and to improve the performance of existing devices [1]. All these characteristics endow molecular electronics a robust technological potential.

A recurrent challenge in this field, however, refers to the establishment of reliable electrical connections to assess the fundamental properties of molecules [8]. The sandwich-like device architecture commonly employed for the electrical characterization of bulky materials is unsuitable because the sublimation of metal electrodes can lead to diffusion-related problems at the molecular scale [2]. To circumvent this issue, different methodologies have been reported for the electrical connection of molecular materials and evaluation of their electrical properties [8]. The scanning tunneling microscopy is the most conventional technique employed to investigate electronic conduction in individual molecules and monolayers. Liquid metal electrodes and the transferring of freestanding electrodes over molecular layers have also been extensively exploited for this purpose [1, 8, 9]. The main limitations regarding such methodologies, however, are related to their lack of compatibility with a real device fabrication technology. This means that devices embedding molecules cannot be manufactured while using such methodologies for the establishment of connections and record of the electrical response.

In this scenario, rolled-up nanomembrane (rNM) technology has emerged as a viable tool to incorporate nanoscale and molecular materials into devices. Such rNMs are straincontrolled thin-films that adopt a self-winding conformation after release from the substrate by the selective removal of a sacrificial layer [10]. The self-curling of metallic nanomembranes is based on the strain release originated on their deposition as thin-films (typically by e-beam evaporation in vacuum) onto a solid substrate. The generated strain is highly dependent on experimental conditions such as the nature of the involved materials, their respective thicknesses, and deposition rates [11, 12]. The etching of a sacrificial layer underneath such thin-films allows their stored elastic energy to be released. The resulting free-standing nanomembrane can adopt different conformations, depending on how such energy release process proceeds. A careful engineering of nanomembranes though allows the production of different structures (e.g., tubes, ribbons, scrolls) [11, 12]. rNMs are typically fabricated using conventional microfabrication techniques and have been applied in batteries [13, 14], sensors [15, 16], superlattices [17, 18], antennas [19], waveguides [20, 21], transistors [22], inductors [23], magnetic structures [24], and biosensors [14, 25]. From the perspective of molecular electronics, one of the most successful uses of rNMs is for the evaluation of the conducting characteristics of ultrathin molecular ensembles [26, 27]. In this case, rNMs are designed to land on top of a molecular layer grown onto a patterned bottom electrode, forming softly-connected metal/ molecular ensemble/metal heterojunctions [27-31]. By using this approach, Merces et al [26] observed an unprecedented conduction mechanism, namely sequential tunneling, to occur in physisorbed molecular ensembles of copper (II) phthalocyanine $(\mathrm{CuPc})$ - a traditional molecular material for electronic devices [30, 32]. Such transport mechanism manifests exclusively in the range of approximately 10-22 nm for CuPc molecular ensembles, being impossible to be verified with a traditional sandwich-like device architecture. Bof Bufon et al [27], using a similar platform, mapped the CuPc charge transport characteristics as a function of the temperature and the applied electric field for $6.5 \mathrm{~nm}$ thick ensembles. Jalil et al [33], exploited the same heterojunction design to fabricate nitrogen dioxide $\left(\mathrm{NO}_{2}\right)$ sensors based on phthalocyanine nanocrystals. Recently, De Oliveira et al probed single-charge effects in phthalocyanine rolled-up heterojunctions containing embedded metal particles in the channel [34]. In all these cases, rNMs were used for accessing the conducting properties of molecular layers. These devices, however, do not allow the evaluation of the charge accumulating properties of molecular materials due to parasitic effects associated with the heterojunction architecture. For this purpose, rNM-based capacitors have been preferred [35]. In this kind of structure, the rNMs form the capacitor's plates, enveloping the molecular material within its structure as part of the dielectric layer. For example, the dielectric properties of chemisorbed, phosphonic acid self-assembled monolayers (SAMs) immobilized on $\mathrm{Al}_{2} \mathrm{O}_{3}$ layers have been characterized as a function of the molecular length (from 6 to 18 carbons) [35]. By using such platform, the dielectric constant of SAMs has been determined.

In this work, we report the design and fabrication rNMbased capacitors for the evaluation of the dielectric properties of physisorbed molecular ensembles. As the molecular material, we chose $\mathrm{CuPc}$ due to its high thermal and chemical stabilities, and well-known morphological, structural and electronic properties [36]. An additional motivation is the lack of experimental data regarding the CuPc dielectric properties at the molecular scale. The similar limitation applies to other molecular semiconductors. According to the literature, the dielectric constant of $\mathrm{CuPc}\left(k_{\mathrm{CuPc}}\right)$ has been reported mostly for $\mu \mathrm{m}$ thick films. For example, Saleh et al [37] obtained $k_{\mathrm{CuPc}} \sim 2.2$ for a $0.4 \mu \mathrm{m}$ thick layer, while Hamam et al [38] characterized $\mathrm{CuPc}$ layers and some derivatives of approximately $50 \mu \mathrm{m}$ thickness, obtaining $k_{\mathrm{CuPc}} \sim 5$.8. Jarosz et al [39] measured $k_{\mathrm{CuPc}}$ values of approximately 3.5 for films thicker than $100 \mathrm{~nm}$. At the molecular scale, $k_{\mathrm{CuPc}}$ has been theoretically predicted though [40-42]. From semi-classical and quantum-mechanical approaches, $k_{\mathrm{CuPc}}$ was calculated for individual $\mathrm{CuPc}$ monomers and vertically stacked oligomers. In both cases, $k_{\mathrm{CuPc}}$ has been found dependent on the molecular orientation [40-42], with in-plane $k_{\mathrm{CuPc}}$ values of 10-20 and perpendicular $k_{\mathrm{CuPc}}$ of approximately 2-3.4. The lack of experimental data on the dielectric constant of CuPc ensembles and other molecular layers can be attributed to the 

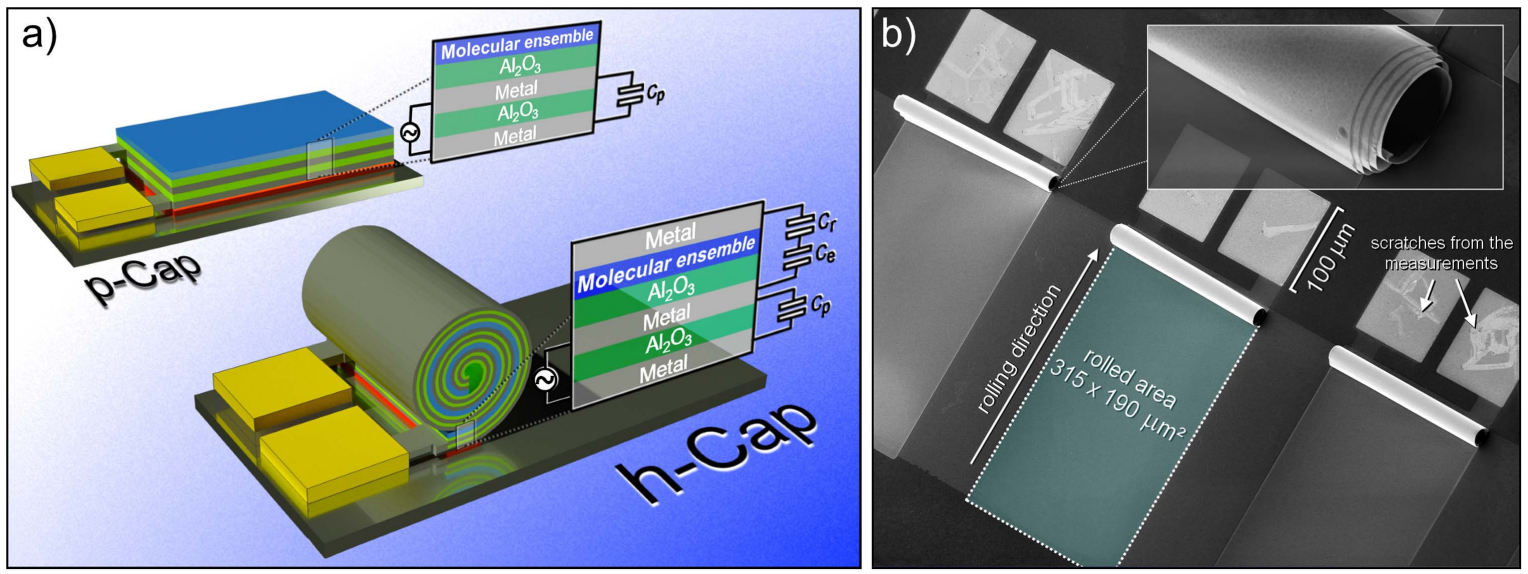

Figure 1. (a) Sketch of the capacitive devices with their respective layer structure and electrical circuit in planar and rolled-up configuration. The hybrid capacitor (h-Cap) is formed after the selective removal of the $\mathrm{GeO}_{x}$ sacrificial layer. The molecular, oxide and metal layers in the devices are, respectively, $\mathrm{CuPc}, \mathrm{Al}_{2} \mathrm{O}_{3}$ and $\mathrm{Au} / \mathrm{Ti} / \mathrm{Cr}$ (except for the inner metal layer which is $\mathrm{Cr}$ ). The parameters $C_{\mathrm{p}}, C_{\mathrm{r}}$, and $C_{\mathrm{e}}$ account for the capacitance of the planar structure, the bare rolled-up device (r-Cap) and the CuPc molecular ensemble. (b) Scanning electron microscopy (SEM) images of a set of three h-Caps. Inset: Magnified image of an h-Cap showing its multiple windings (five).

challenge in connecting such ultrathin structures in a twoelectrode, parallel-plate capacitor geometry for capacitance measurements. Here, hybrid capacitors (h-Caps) based on metallic rNMs, $\mathrm{Al}_{2} \mathrm{O}_{3}$ dielectric and $\mathrm{CuPc}$ molecular ensembles of different thicknesses $(5-50 \mathrm{~nm})$ have been fabricated and investigated. The device architecture is designed to envelop the $\mathrm{CuPc}$ layer with rNMs, providing a soft and reliable electrical connection with the molecular ensemble. An $\mathrm{Al}_{2} \mathrm{O}_{3}$ dielectric layer is used together with the $\mathrm{CuPc}$ ensemble to prevent short-circuit through the capacitor plates (see figure 1(a)). From the analysis and modeling of the h-Cap response, the dielectric contribution of the embedded molecular ensembles was obtained, allowing the successful calculation of CuPc dielectric constant $\left(k_{\mathrm{CuPc}}=4.5 \pm 0.5\right)$. The reported strategy is a viable route for the dielectric characterization of ultrathin, physisorbed molecular layers, with no need of expensive apparatus, and in consonance with a real device technology.

\section{Materials and methods}

The h-Cap fabrication is based on the spontaneous roll-up of a parallel-plate planar capacitor ( $\mathrm{p}$-Cap) having strained nanomembranes as the metal plates and aluminum oxide $\left(\mathrm{Al}_{2} \mathrm{O}_{3}\right)$ as the dielectric, as illustrated in figure 1(a). The whole device structure is assembled onto a $\mathrm{GeO}_{x}$ sacrificial layer, which after selective removal allows the nanomembrane to roll-up (figure 1(b)). Before this process, however, an additional $\mathrm{Al}_{2} \mathrm{O}_{3}$ layer is grown onto the p-Cap to electrically insulate the multiple windings of the final rolled-up device. The $\mathrm{CuPc}$ molecular ensemble is deposited as the p-Cap outermost layer. The h-Caps are designed to envelop the molecular layer, with electrical connections provided by mechanically soft rNM capacitor plates. Details of the h-Cap fabrication processes, materials and methods used are presented in the following.
The h-Caps were fabricated on $9 \mathrm{~mm} \times 9 \mathrm{~mm} \mathrm{Si}(100)$ substrates covered with $1 \mu \mathrm{m}$ thick $\mathrm{SiO}_{2}$ top layer. The whole device fabrication relies on conventional optical photolithography and thin-film deposition methods. Here, we employed thermal evaporation (e-beam) for the deposition of metallic layers (viz. $\mathrm{Ge}, \mathrm{Au}, \mathrm{Ti}$, and $\mathrm{Cr}$ ) and (resistive filament) for the deposition of the CuPc molecular ensemble. In all cases, the material deposition was carried out at room temperature, at a rate of $2 \AA \mathrm{s}^{-1}$ and a vacuum pressure of approximately $10^{-6}$ Torr. A quartz crystal microbalance was used to monitor the respective thicknesses of the deposited layers in the deposition chamber. The important factors that led to the choice for $\mathrm{Ge}, \mathrm{Au}, \mathrm{Ti}$, and $\mathrm{Cr}$ are described in the supporting information, available online at stacks.iop.org/ NANO/29/265201/mmedia. $\mathrm{Al}_{2} \mathrm{O}_{3}$ layers were obtained by atomic layer deposition (ALD) method to achieve films with excellent uniformity, conformity and controlled thickness [43]. The option for $\mathrm{Al}_{2} \mathrm{O}_{3}$ relies on its high dielectric constant ( 9) [44] and wide bandgap ( 2.7 eV) [45], which provide small leakage currents and dielectric losses suitable for capacitive devices. The $\mathrm{Al}_{2} \mathrm{O}_{3} \mathrm{ALD}$ films were grown in an Oxford OpAL reaction chamber using as precursors trimethylaluminum 97\% (TMA), from Sigma-Aldrich, and $\mathrm{H}_{2} \mathrm{O}$. The temperature of the sample stage was kept at $150{ }^{\circ} \mathrm{C}$ during the $\mathrm{Al}_{2} \mathrm{O}_{3}$ deposition. The chamber pressure was set between 120 and 260 mbar, and argon was used as the carrier/purging gas at a flow from 30 to $100 \mathrm{sccm}$. The deposition time for $10 \mathrm{~nm}$ of the $\mathrm{Al}_{2} \mathrm{O}_{3}$ layer was $40 \mathrm{~min}$ at a growth rate of $1.5 \AA /$ cycle.

The device fabrication starts with the thermal deposition of a $20 \mathrm{~nm}$ thick $\mathrm{Ge}$ layer onto the $\mathrm{Si} / \mathrm{SiO}_{2}$ substrate. Afterward, a heat treatment at $60{ }^{\circ} \mathrm{C}$ is carried out for $17 \mathrm{~h}$ to oxidize $\mathrm{Ge}$ and form $\mathrm{GeO}_{x}$, which can be removed in water during the rolled-up procedure [35]. The second step consists in the deposition of the capacitor bottom plate. Here, we used a strained layer formed by sequentially deposited $\mathrm{Au}(5 \mathrm{~nm})$, $\mathrm{Ti}(10 \mathrm{~nm})$, and $\mathrm{Cr}(10 \mathrm{~nm})$. The weak adherence of $\mathrm{Au}$ on 
$\mathrm{GeO}_{x}$ facilitates the detachment of the strained capacitor plate from the sacrificial layer during the roll-up procedure. An $\mathrm{Al}_{2} \mathrm{O}_{3}$ dielectric layer $(10 \mathrm{~nm})$ was deposited onto the $\mathrm{p}$-Cap bottom plate, followed by the deposition of $10 \mathrm{~nm}$ of $\mathrm{Cr}$ as the capacitor's top plate. As previously mentioned, an additional $10 \mathrm{~nm} \mathrm{Al}_{2} \mathrm{O}_{3}$ layer is deposited onto the top plate to electrically insulate the multiple windings of the final rolled-up capacitor. The device connecting terminals were produced by initially clearing the contact areas with $1 \% \mathrm{v} / \mathrm{v}$ hydrofluoric acid (HF) aqueous solution, followed by the deposition of $\mathrm{Cr}$ and $\mathrm{Au}(20 / 40 \mathrm{~nm})$ electrical pads. HF corrosion was also used to open a trench over the upper region of the p-Cap to facilitate the access of water and dissolution of $\mathrm{GeO}_{x}$ during the roll-up procedure. All critical regions of the device were protected from corrosion by a thin photoresist layer. Finally, the $\mathrm{CuPc}$ molecular layer was deposited from powder (576.08 $\mathrm{g} \mathrm{mol}^{-1}$, purchased from Alfa Aesar) over the planar device, ready to initiate the roll-up procedure. In the supporting information, a sketch with all device fabrication steps is shown (figure S1), along with an optical image of a set containing twenty-four devices that illustrates the reproducibility of the method (figure S2).

The roll-up procedure is carried out by inserting the planar devices in $1 \% \mathrm{v} / \mathrm{v} \mathrm{H}_{2} \mathrm{O}_{2}: \mathrm{H}_{2} \mathrm{O}$ solution for the $\mathrm{GeO}_{x}$ dissolution. The formation of rolled-up h-Caps takes approximately $60 \mathrm{~min}$. During this process, the elastic energy stored in the strained nanomembranes is controllably released, leading the whole structure to curl up. The geometrical control of the h-Caps regarding the number of windings and the tube diameter is achieved by adjusting the deposition rate and thickness of the strained layers, and the extension of the rolling path [35, 46]. Here, five sets of h-Caps with an average of five turns were fabricated (figure 1(b)). For each set of devices, CuPc layers of different thicknesses $(5-50 \mathrm{~nm})$ were deposited. The respective thicknesses of the molecular ensembles were monitored by atomic force microscopy (AFM), as illustrated in figure S3. Rolled-up capacitors absent of $\mathrm{CuPc}$, here named r-Caps, were also fabricated for comparison.

For the determination of the CuPc dielectric properties using h-Caps, ac electrical measurements, namely capacitance versus frequency $(C-f)$ and impedance phase angle versus frequency (theta- $f$ ), were performed. The electrical measurements were carried out using an MPS150 Cascade Microtech probe station positioned into a Faraday cage and coupled with a Keysight E4980A impedance instrument. $C-f$ and theta- $f$ measurements were performed employing a sinewave voltage stimulus of $100 \mathrm{mV}$ amplitude, with frequencies spanning from $100 \mathrm{~Hz}$ to $2 \mathrm{MHz}$. The ac response of the devices was fitted based on their equivalent circuit representation using Zview ${ }^{\circledR}$ software. Additional dc electrical characterization, namely current $(I)$-electric field $(E)$ measurements, were performed using a Keithley 4200 SCS analyzer. As the diameter of the rolled-up device is considerably larger than its thickness ( $\mu \mathrm{m}$ versus $\mathrm{nm}$ ), we can determine the applied $E$ considering the parallel-plate capacitor approximation, i.e., $E=V / \ell$, where $\ell$ is the sample thickness and $V$ the applied voltage. Before the electrical characterizations, the devices were stored in the vacuum $\left(10^{-2}\right.$ Torr) for at least $2 \mathrm{~h}$ to remove adventitious contaminants from the roll-up procedure. All electrical measurements were performed in the dark, and in a laboratory environment.

\section{Results and discussion}

Figure 1(b) shows a scanning electron microscopy (SEM) image of three h-Caps on the same substrate. The devices present in average 5 windings, with an external diameter $(d)$ of approximately $20 \pm 3 \mu \mathrm{m}$. This corresponds to a decrease of the device area from $6 \times 10^{-8} \mathrm{~m}^{2}$ in a planar configuration to $4 \times 10^{-9} \mathrm{~m}^{2}$, a reduction of $>90 \%$ of the capacitor footprint. Such an ultracompact architecture is advantageous to envelop the molecular layer, preserving its integrity by adventitious external agents that may affect the properties of $\mathrm{CuPc}$ during the electrical measurements, e.g., humidity or atmospheric gases [47]. The most appealing feature of such rolled-up devices, however, lies on the soft electrical contact established between the nanomembrane capacitor plates and the molecular ensemble, allowing the evaluation of the dielectric properties of ultrathin layers.

Figure 2 illustrates the measured capacitance $\left(C_{\mathrm{M}}\right)$ as a function of frequency for an h-Cap containing $15 \mathrm{~nm}$ of CuPc. The responses of the bare devices (viz. metal $/ \mathrm{Al}_{2} \mathrm{O}_{3} /$ metal capacitor), in both planar (p-Cap) and rolled-up (r-Cap) configuration, were added for comparison. The curves in figure 2 correspond to data obtained from five similar devices with error bars associated with standard deviation values of $C_{\mathrm{M}}$. The respective impedance phase angle (theta) is exhibited inset. From $C_{\mathrm{M}}-f$ plots, capacitance plateaus with phase angles of $-90^{\circ}$, for a wide range of frequencies (from 100 to $10^{5} \mathrm{~Hz}$ ), are observed for all types of devices. These characteristics corroborate the capacitive behavior of the devices in both planar and rolled-up geometries. A comparison between the bare $\mathrm{p}$ - and r-Caps indicates the expected capacitance increase when the planar structure adopts a tubular conformation [35]. Such a capacitance increase occurs due to the formation of an additional capacitor $\left(C_{\mathrm{r}}\right)$ related to the rolled-up geometry that is connected in parallel with the planar one $\left(C_{\mathrm{p}}\right)$. Here, $C_{\mathrm{M}}$ increases from $347 \pm 1 \mathrm{pF}$ in p-Caps to $486 \pm 2 \mathrm{pF}$ in r-Caps for values taken at $1 \mathrm{kHz}$. Bof Bufon et al [35] showed the capacitance of rolled-up capacitors depends on the number of windings $(N)$, through the relationship $C_{\mathrm{r}} / C_{\mathrm{p}}=2-(1 / N)$. Here, as the fabricated rolled-up capacitors have an average of 5 turns, then $C_{\mathrm{r}} / C_{\mathrm{p}}$ should be equal the 1.8. For the presented devices this relation was found 1.4 though. Such a difference is associated with voids between the windings caused by loose turns of the rNMs [46]. From the SEM images (inset of figure 1(b)), this seems to be the case for the presented devices. The presence of voids, however, does not prevent the dielectric characterization of ultrathin $\mathrm{CuPc}$ layers $(<30 \mathrm{~nm})$, as an effective connection between the electrode plates and the molecular ensemble can be achieved. For thicker layers $(30-50 \mathrm{~nm})$, the compactness of h-Caps is compromised, which does not 


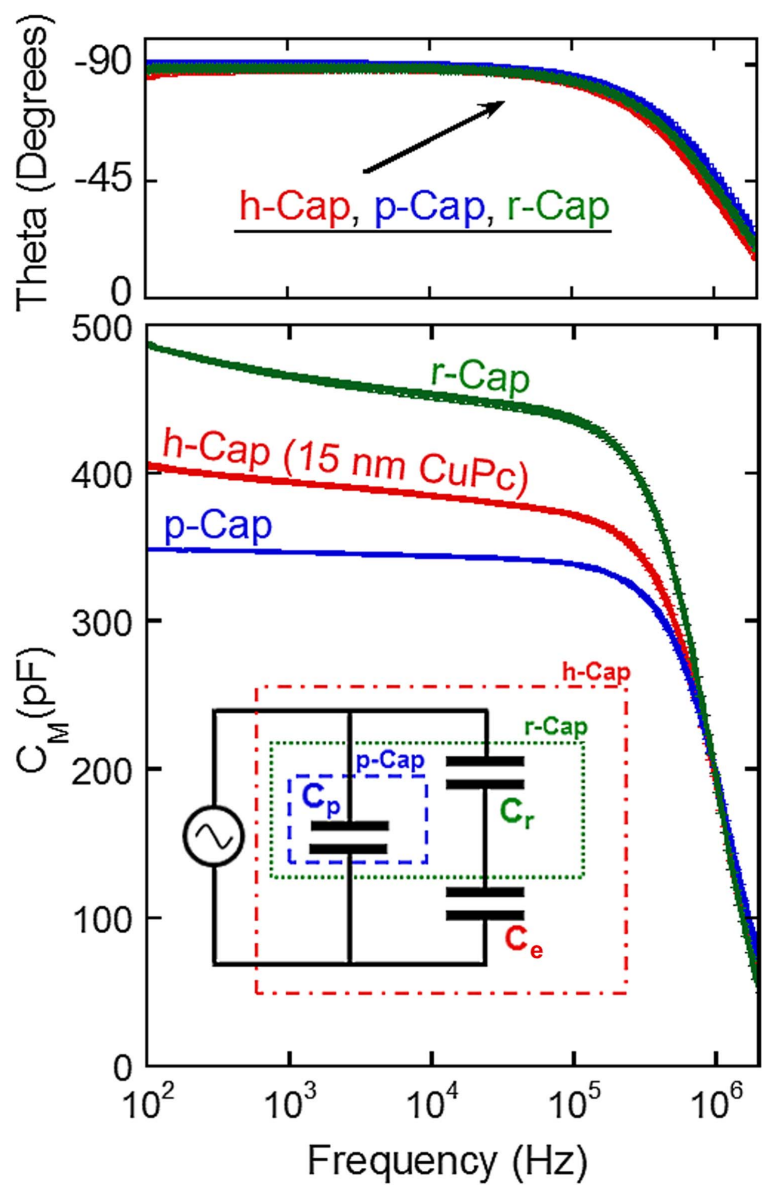

Figure 2. Impedance phase (theta) and measured capacitance $\left(C_{\mathrm{M}}\right)$ as a function of frequency for the planar capacitor (p-Cap), the bare rolled-up device (r-Cap), and the hybrid rolled-up capacitor (h-Cap) having $15 \mathrm{~nm}$ thick of $\mathrm{CuPc}$ layer. The error bars correspond to variations of data measured for five similar devices (error bars are mainly noticeable at high frequencies). Inset: h-Cap equivalent circuit with the individual capacitance contributions of the planar geometry $\left(C_{\mathrm{p}}\right)$, the bare rolled-up structure $\left(C_{\mathrm{r}}\right)$, and the $\mathrm{CuPc}$ molecular ensemble $\left(C_{\mathrm{e}}\right)$.

hamper the capacitance curves to be obtained but may lead to inconsistencies in the interpretation of the molecular dielectric properties, as further discussed in detail.

The incorporation of the CuPc molecular ensemble in the rolled-up capacitors reduces $C_{\mathrm{M}}$ from the values exhibited by bare r-Caps. Here, a decrease from $468 \pm 2 \mathrm{pF}$ to $395 \pm 2 \mathrm{pF}$ is observed at $1 \mathrm{kHz}$. As the CuPc molecular ensemble is deposited atop the $\mathrm{Al}_{2} \mathrm{O}_{3}$ layer in h-Caps, this can be schematically seen as the inclusion of an additional capacitor $\left(C_{\mathrm{e}}\right)$ in series with $C_{\mathrm{r}}$ to account for the CuPc dielectric properties. In this sense, $C_{\mathrm{M}}$ is reduced due to a new component in the circuit (figures 1(a) and 2, inset). The incorporation of the $\mathrm{CuPc}$ layer onto p-Caps did not cause any appreciable changes in the low-frequency $C_{\mathrm{M}}$ when compared with their bare counterparts, as shown in figure S4.

To characterize the dielectric properties of $\mathrm{CuPc}$, in specific $k_{\mathrm{CuPc}}$, we investigated the response of h-Caps having different thicknesses $\left(t_{\mathrm{CuPc}}\right)$. Figure 3(a) shows $C_{\mathrm{M}}$ for devices presenting $t_{\mathrm{CuPc}}$ varying from 5 to $50 \mathrm{~nm}$. The data corresponds to the mean capacitance values taken from five similar devices, with error bars associated with the standard deviation of $C_{\mathrm{M}}$. From this plot, one can observe that $C_{\mathrm{M}}$ (at a given frequency, e.g., $1 \mathrm{kHz}$ ) substantially decreases as $t_{\mathrm{CuPc}}$ increases, mainly from 5 to $20 \mathrm{~nm}$. For thicker layers, $C_{\mathrm{M}}$ oscillates around $414 \pm 4 \mathrm{pF}$, as a further decrease would be expected with the increase of $t_{\mathrm{CuPc}}$. The reason for $C_{\mathrm{M}}$ not following such a trend is based on the compactness of h-Caps. For small thicknesses $(<30 \mathrm{~nm})$, thin $\mathrm{CuPc}$ molecular ensembles exhibit low roughness values $(1.2 \pm 0.3 \mathrm{~nm})$, as indicated by AFM measurements in figure S3. In this case, the ultrathin molecular layer follows the topography of $\mathrm{Al}_{2} \mathrm{O}_{3}$ dielectric, which presents rms roughness values of approximately $1.1 \pm 0.3 \mathrm{~nm}$. Thus, the compactness of h-Caps is not affected, and the devices exhibit the typical shape shown in figure 1(b) (inset). The incorporation of ultrathin molecular layers in rNM capacitors is feasible indeed, as previously demonstrated for SAMs $(\sim 0.8-2.0 \mathrm{~nm})$ [35]. However, for thicker layers (CuPc ensembles $>30 \mathrm{~nm}$ ), the h-Cap compactness is compromised. The higher roughness values for such $\mathrm{CuPc}$ ensembles $(3.8 \pm 0.3 \mathrm{~nm})$ contribute to such lack of compactness by reducing the electrode/molecule contact area. Thus, for thick molecular layers, $C_{\mathrm{M}}$ is substantially affected (figures 3(a) and 4(a)).

The leakage current of h-Caps has also been evaluated. Figure 3(b) shows $I-E$ curves for all types of devices, namely p-Caps, bare r-Caps, and h-Caps. The $I-E$ response of h-Caps with all evaluated thicknesses $(5-50 \mathrm{~nm})$ fall in the hatched area. For all curves, we determined the electric breakdown $\left(E_{\mathrm{B}}\right)$, i.e., the electric field where the current increases abruptly. These values are highlighted in the respective curves. Here, we observed high $E_{\mathrm{B}}\left(7.0 \pm 0.1 \mathrm{MV} \mathrm{cm}^{-1}\right)$ for p-Caps, in agreement with the literature for devices employing thin $\mathrm{Al}_{2} \mathrm{O}_{3}$ dielectric layers [43]. For r-Caps, $E_{\mathrm{B}}$ was found $2.1 \pm 0.4 \mathrm{MV} \mathrm{cm}^{-1}$, being considerably smaller than the values found for p-Caps. The decrease of $E_{\mathrm{B}}$ can be attributed to the formation of microcracks in the $\mathrm{Al}_{2} \mathrm{O}_{3}$ layer [45, 48], facilitating the current flow through such defects. The origin of such oxide cracks is related to the conformation adopted by the device when the planar structure is curled up. For h-Caps, $E_{\mathrm{B}}$ varied from $3.1 \pm 0.3$ to $4.2 \pm 0.3 \mathrm{MV} \mathrm{cm}^{-1}$ (hatched area, figure 3(b)), superior to the values exhibited by bare r-Caps. Thus, the CuPc molecular ensembles act as leakage current suppressors in such devices.

Here, the aim is to use h-Caps to experimentally determine the dielectric properties molecular ensembles, in special $k_{\mathrm{CuPc}}$. For this purpose, firstly we determined the equivalent capacitance $\left(C_{\mathrm{h}}\right)$ of the series branch of the h-Cap circuit which contains information of the molecular layer $\left(C_{\mathrm{e}}\right)$. From the equivalent circuit, we have $C_{\mathrm{h}}=C_{\mathrm{M}}-C_{\mathrm{p}}$. Thus, from the $C_{\mathrm{M}}$ values at $1 \mathrm{kHz}$ for all analyzed thicknesses (figure 3(a)), we can express $C_{\mathrm{h}}$ as a function of $t_{\mathrm{CuPc}}$, as illustrated in figure 4(a).

For small CuPc thicknesses $(\leqslant 20 \mathrm{~nm})$, the experimental data can be represented by the series combination of two ideal capacitors, namely $C_{\mathrm{r}}$ and $C_{\mathrm{e}}$. For thicker layers, however, it is necessary to utilize a non-ideal capacitor component, namely constant phase element (CPE), to represent $C_{\mathrm{e}}$. The $\mathrm{CPE}$ is commonly used to represent irregularities in device 

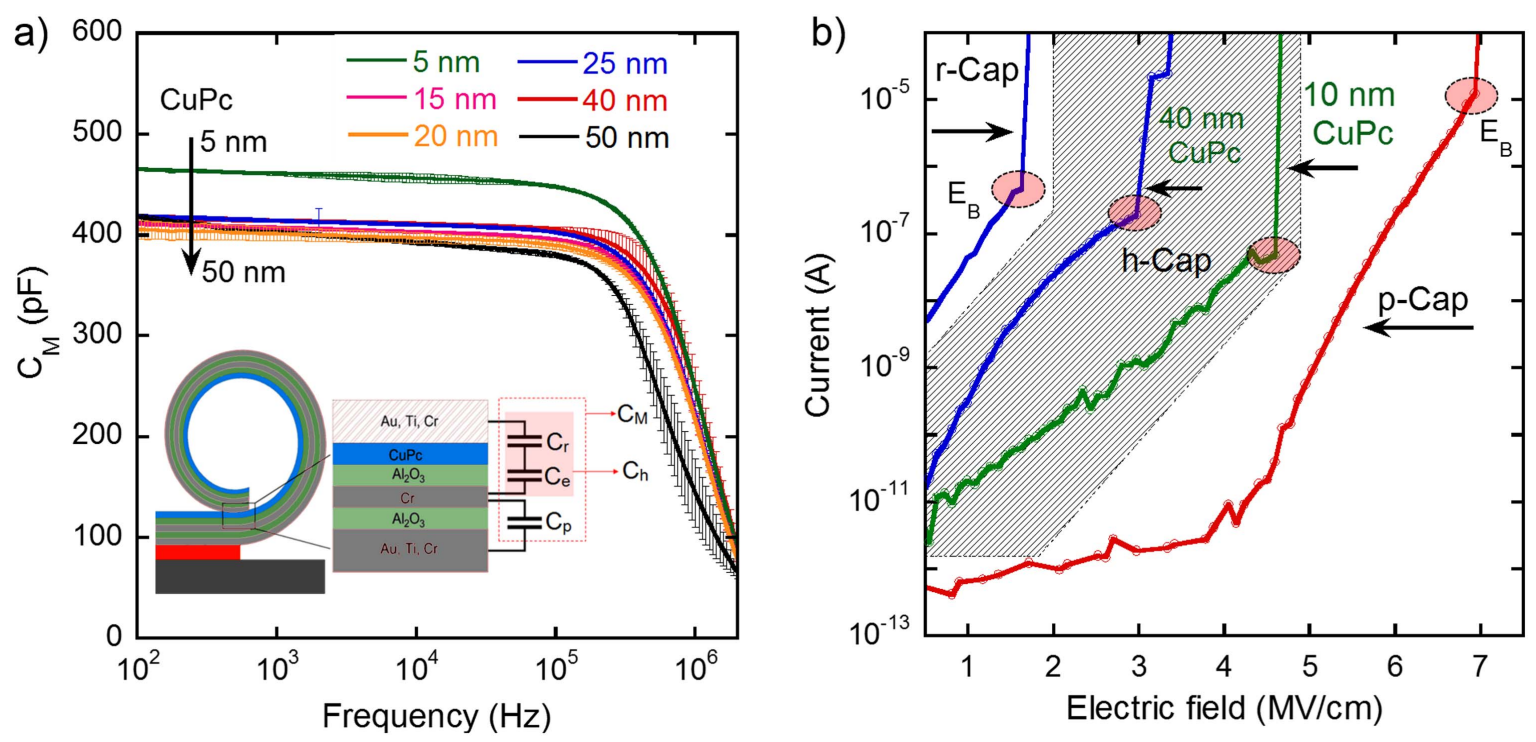

Figure 3. (a) $C_{\mathrm{M}^{-}}-f$ characteristics for h-Caps having CuPc layers with different thicknesses (from 5 to $50 \mathrm{~nm}$ ). The error bars correspond to standard deviation values calculated from five similar devices. Inset: h-Cap architecture and respective equivalent circuit. (b) Current-electric field $(I-E)$ for p-Caps, r-Caps, and h-Caps (for 10 and $40 \mathrm{~nm}$ CuPc layers). The $I-E$ response of h-Caps with all evaluated thicknesses fall into the hatched area. The corresponding electric breakdown $\left(E_{\mathrm{B}}\right)$ are highlighted in each curve. As the p-Cap is rolled-up (r-Cap), $E_{\mathrm{B}}$ decreases from $7.0 \pm 0.1$ to $2.1 \pm 0.4 \mathrm{MV} \mathrm{cm}^{-1}$ due to the formation of microcracks in the $\mathrm{Al}_{2} \mathrm{O}_{3}$ layer. The incorporation of CuPc molecular ensembles behaves as a leakage current suppressor, leading to slightly higher $E_{\mathrm{B}}$ values.

geometry (e.g., surface imperfections) that deviate the measured capacitance from the ideal behavior [49]. In our case, as the compactness of h-Caps is reported to be affected for thick CuPc ensembles $(>30 \mathrm{~nm})$, the use of CPE is appropriate, and modeling shows good agreement with the data, as illustrated in figure 4(a). Details on the equivalent circuit modeling used here are given in the supporting information (figures S5 and S6). To extract $k_{\mathrm{CuPc}}$, the thickness range 5-20 nm was used for simplicity. In this range, $C_{\mathrm{h}}$ is given by equation (1):

$$
\frac{1}{C_{\mathrm{h}}}=\frac{t_{\mathrm{ox}}}{k_{\mathrm{ox}} \cdot \varepsilon_{0} \cdot A}+\frac{1}{k_{\mathrm{CuPc}} \cdot \varepsilon_{0} \cdot A} \cdot t_{\mathrm{CuPc}}
$$

whereas $t_{\mathrm{ox}}$ and $k_{\mathrm{Ox}}$ are, respectively, the thickness and dielectric constant of the $\mathrm{Al}_{2} \mathrm{O}_{3}$ layer, $\varepsilon_{0}$ is the free space permittivity $\left(8.85 \times 10^{-12} \mathrm{~F} \mathrm{~m}^{-1}\right)$, and $A$ is the device contact area. The values of $k_{\mathrm{ox}}$ and $t_{\mathrm{ox}}$ were experimentally extracted from the characterization of p-Caps, being respectively $6.7 \pm 0.1$ and $10.0 \pm 0.3 \mathrm{~nm}$. The contact area $(A)$ was obtained from the electrical measurements of bare r-Caps, resulting in $A=(2.4 \pm 0.2) \times 10^{-8} \mathrm{~m}^{2}$. The far right side of equation (1) accounts for $C_{\mathrm{e}}$, which contains the parameter of interest $\left(k_{\mathrm{CuPc}}\right)$. Figure 4(b) shows the inverse of $C_{\mathrm{e}}$ as a function of $t_{\mathrm{CuPc}}$, where $k_{\mathrm{CuPc}}$ was calculated using the method of ordinary least squares. For the range of $t_{\mathrm{CuPc}}$ analyzed $(5-20 \mathrm{~nm}), k_{\mathrm{CuPc}}$ was found $4.5 \pm 0.5$, slightly higher than the values reported for $\mu \mathrm{m}$ thick CuPc layers $[38,39]$. Considering that $k_{\mathrm{CuPc}}$ depends on the molecular orientation [40-42], whereas the in-plane $k_{\mathrm{CuPc}}$ is 10-20 and the perpendicular one is much lower (approximately 2-3.4), our values suggest a mild contribution of the in-plane field-induced dipole of $\mathrm{CuPc}$ to the measured $k_{\mathrm{CuPc}}$ values. Thus, we may infer that CuPc molecules on the electrode surface are not in a perfect lying configuration in respect to the vertical applied electric field, but partially oriented in standing up direction. The literature reports indeed such a partially standing orientation of $\mathrm{CuPc}$ molecules deposited onto amorphous oxide surfaces such as $\mathrm{SiO}_{2}$ [50], and $\mathrm{Al}_{2} \mathrm{O}_{3}$. The exact molecular orientation, however, is difficult to determine for ultrathin layers. Finally, to validate our experimental paradigm, we have fabricated and evaluated h-Caps containing cobalt (II) phthalocyanine $(\mathrm{CoPc})$ molecular ensembles which have great potential for spintronics [51, 52]. The devices were fabricated and measured following the same experimental procedures reported for $\mathrm{CuPc}$ devices. From the capacitance response of CoPc h-Caps (figure S7), the dielectric constant $\left(k_{\mathrm{CoPc}}\right)$ was found $2.7 \pm 0.4$, also in agreement with the literature [53]. Our findings thus demonstrate the dielectric properties of molecular layers resemble those typically found in thin-films widely employed in electronic devices, with a mild contribution of the molecular orientation. Therefore, novel developments in the field of molecular electronics can benefit from the vast literature reporting the dielectric properties of such materials. Finally, the reported h-Cap technology can be used to determine the dielectric properties of thin-films of any nature, as long as these films are compatible with the fabrication methodology employed here, mainly regarding the chemical stability of such films in $\mathrm{H}_{2} \mathrm{O}$ or in the $\mathrm{H}_{2} \mathrm{O}_{2}(1 \% \mathrm{v} / \mathrm{v}) / \mathrm{H}_{2} \mathrm{O}$ roll-up solution.

\section{Conclusion}

In this work, we reported on the evaluation of the dielectric properties of ultrathin molecular ensembles integrated into hybrid capacitive devices with strong potential for molecular electronics. The devices consisted of ultracompact capacitors with strained nanomembranes as the metal plates and an $\mathrm{Al}_{2} \mathrm{O}_{3}$ layer as the dielectric, in the so-called rolled-up 
a)

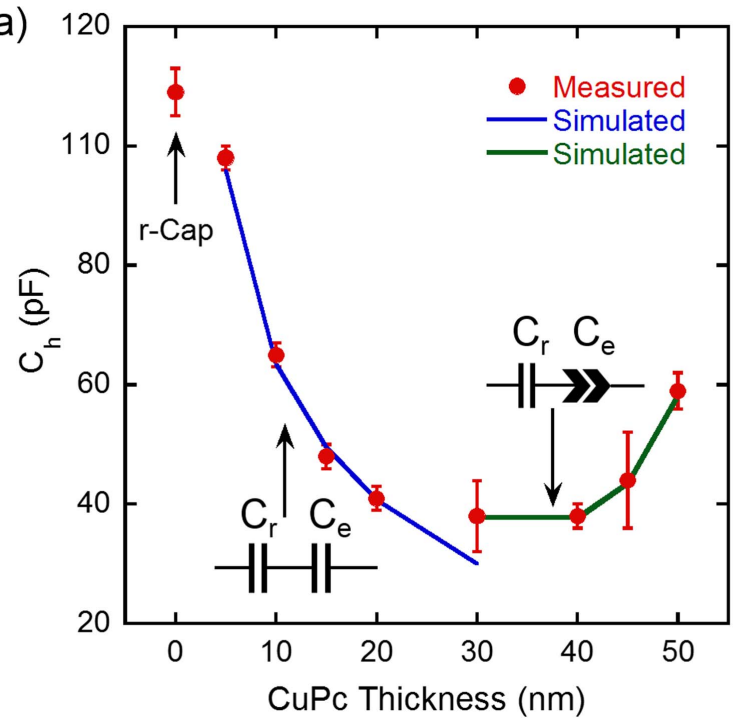

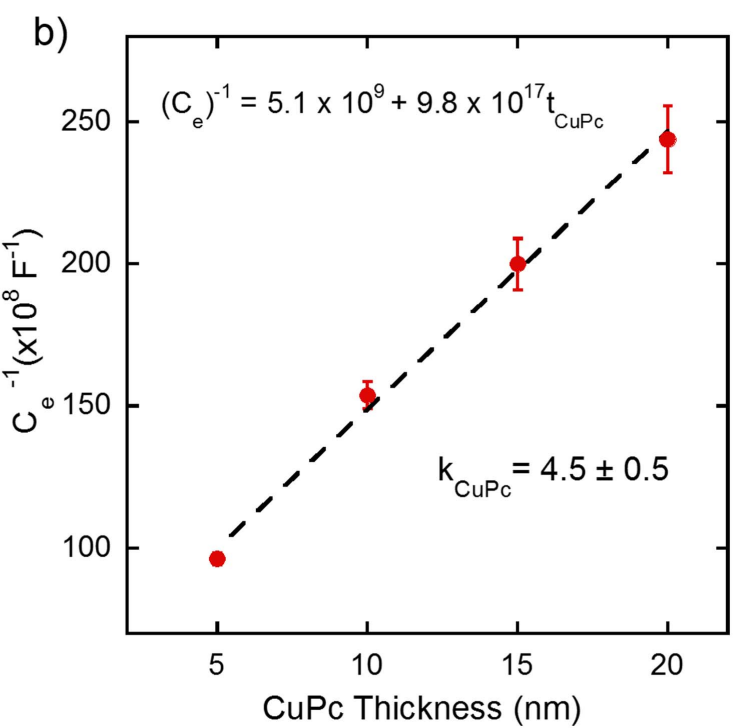

Figure 4. (a) $C_{\mathrm{h}}$ (at $\left.1 \mathrm{kHz}\right)$ as a function of the CuPc thickness $\left(t_{\mathrm{CuPc}}\right)$. For small thicknesses $(5-20 \mathrm{~nm}), C_{\mathrm{h}}$ decreases as $t_{\mathrm{CuPc}}$ increases. The data can be represented by a series association of $C_{\mathrm{r}}$ and $C_{\mathrm{e}}$, considering two ideal capacitors. For thicker CuPc layers (30-50 nm), the data is better represented by the series association of an ideal capacitor and a CPE. The capacitance of the base r-Cap is also shown. (b) $C_{\mathrm{e}}{ }^{-1}$ as a function of $t_{\mathrm{CuPc}}$ in the range of 5-20 nm. The curve slope gives $k_{\mathrm{CuPc}}\left(R^{2}=0.996\right.$ for the linear fit $)$. The error bars in both plots correspond to standard deviation values obtained considering five similar devices.

architecture. The capacitor nanomembrane plates provide a mechanically soft, yet robust electrical connection with the embedded molecular ensembles, while the $\mathrm{Al}_{2} \mathrm{O}_{3}$ layer prevents short-circuit from occurring. As the molecular material, physisorbed copper (II) phthalocyanine $(\mathrm{CuPc})$ ensembles were used, as experimental data on the $\mathrm{CuPc}$ dielectric constant at the molecular scale has not been reported. The fabricated h-Caps exhibited good compactness (diameter of approximately $20 \pm 3 \mu \mathrm{m}$ ) for molecular ensembles with a thickness ranging from 5 to $20 \mathrm{~nm}$, which allow the dielectric characterization of CuPc. In this range, the dielectric constant of the molecular material was found $k_{\mathrm{CuPc}}=4.5 \pm 0.5$. For thicker layers (up to $50 \mathrm{~nm}$ ), the compactness of the h-Caps is compromised, leading to inconsistencies in the capacitance data. We validated our experimental paradigm by measuring the dielectric properties of cobalt (II) phthalocyanine ensembles, which have significant appeal for molecular spintronics. For both molecular ensembles, the measured dielectric constant agrees with data in the literature for their thin-film counterparts ( $\mu \mathrm{m}$ thick layers). We thus demonstrate the dielectric properties of molecular layers are equivalent to those found in bulky layers. The reported h-Cap is a reliable strategy for the characterization of the molecular dielectric properties using a real device technology.

\section{Acknowledgments}

The authors acknowledge CAPES, CNPq (Project 483550/ 2013-2), and FAPESP (Project 2014/25979-2) for the financial support. We would like to thank the National Center for Research in Energy and Materials (CNPEM) and Brazilian Nanotechnology National Laboratory (LNNano).

\section{Author contribution}

PAP fabricated and evaluated the devices, performed data analysis, and wrote the manuscript. RMLS fabricated devices and performed measurements, RFO and LM contributed to data discussion and the manuscript writing, CCBB supervised the experiments, discussed the results, contributed to the manuscript writing, and led the work.

\section{ORCID iDs}

Rafael F de Oliveira (i) https://orcid.org/0000-00018980-3587

Leandro Merces 수 https://orcid.org/0000-0002-6202-9824

\section{References}

[1] Xiang D et al 2016 Molecular-scale electronics: from concept to function Chem. Rev. 116 4318-440

[2] Vilan A, Aswal D and Cahen D 2017 Large-area, ensemble molecular electronics: motivation and challenges Chem. Rev. 117 4248-86

[3] Elsaidi S K, Mohamed M H, Banerjee D and Thallapally P K 2018 Flexibility in metal-organic frameworks: a fundamental understanding Coord. Chem. Rev. 358 125-52

[4] Srivastava A and Khan M S 2018 First principle study of single electron transistor based on metal-organic complex of dibenzothiophene Org. Electron. 53 227-34

[5] Baran D, Ashraf R S, Hanifi D A, Abdelsamie M, Gasparini N, Röhr J A, Holliday S, Wadsworth A, Lockett S and Neophytou M 2017 Reducing the efficiency-stability-cost gap of organic photovoltaics with highly efficient and stable small molecule acceptor ternary solar cells Nat. Mater. 16 363-9 
[6] Li W, Torres D, Díaz R, Wang Z, Wu C, Wang C, Lin Wang Z and Sepúlveda N 2017 Nanogenerator-based dual-functional and self-powered thin patch loudspeaker or microphone for flexible electronics Nat. Commun. 815310

[7] Thoss M and Evers F 2018 Perspective: theory of quantum transport in molecular junctions J. Chem. Phys. 148030901

[8] Haick H and Cahen D 2008 Making contact: connecting molecules electrically to the macroscopic world Prog. Surf. Sci. 83 217-61

[9] Jeong H, Kim D, Xiang D and Lee T 2017 High-yield functional molecular electronic devices ACS Nano 7 6511-48

[10] Prinz V Y, Naumova E V, Golod S V, Seleznev V A, Bocharov A A and Kubarev V V 2017 Terahertz metamaterials and systems based on rolled-up 3D elements: designs, technological approaches, and properties Sci. Rep. 7 43334

[11] Nikishkov G P 2003 Curvature estimation for multilayer hinged structures with initial strains J. Appl. Phys. 945333

[12] Grundmann M 2003 Nanoscroll formation from strained layer heterostructures Appl. Phys. Lett. 83 2444-6

[13] Deng J, Ji H, Yan C, Zhang J, Si W, Baunack S, Oswald S, Mei Y and Schmidt O G 2013 Naturally rolled-up C/Si/C trilayer nanomembranes as stable anodes for lithium-ion batteries with remarkable cycling performance Angew. Chem., Int. Ed. Engl. 52 2326-30

[14] Liu B, Yan C, Si W, Sun X, Lu X, Ansorge-Schumacher M and Schmidt O G 2018 Ultralongdischarge-time biobattery based on immobilized enzymes in bilayer rolled-up enzymatic nanomembranes Small 14 1704221

[15] Vervacke C, Bufon C C B, Thurmer D J and Schmidt O G 2014 Three-dimensional chemical sensors based on rolledup hybrid nanomembranes RSC Adv. 4 9723-9

[16] Jalil A R, Chang H, Bandari V K, Robaschik P, Zhang J, Siles P F, Li G, Bürger D, Grimm D and Liu X 2016 Fully integrated organic nanocrystal diode as high performance room temperature $\mathrm{NO}_{2}$ sensor Adv. Mater. 28 2971-7

[17] Grimm D, Wilson R B, Teshome B, Gorantla S, Rümmeli M H, Bublat T, Zallo E, Li G, Cahill D G and Schmidt O G 2014 Thermal conductivity of mechanically joined semiconducting/metal nanomembrane superlattices Nano Lett. 14 2387-93

[18] Li G, Grimm D, Engemaier V, Lösch S, Manga K, Bandari V K, Zhu F and Schmidt O G 2016 Hybrid semiconductor/metal nanomembrane superlattices for thermoelectric application Phys. Status Solidi a 213 620-5

[19] Karnaushenko D, Münzenrieder N, Karnaushenko D D, Koch B, Meyer A K, Baunack S, Petti L, Tröster G, Makarov D and Schmidt O G 2015 Biomimetic microelectronics for regenerative neuronal cuff implants Adv. Mater. 27 6797-805

[20] Huang G and Mei Y 2017 Electromagnetic wave propagation in a rolled-up tubular microcavity J. Mater. Chem. C 5 2758-70

[21] Yu X, Arbabi E, Goddard L L, Li X and Chen X 2015 Monolithically integrated self-rolled-up microtube-based vertical coupler for three-dimensional photonic integration Appl. Phys. Lett. 107031102

[22] Grimm D, Bof Bufon C C, Deneke C, Atkinson P, Thurmer D J, Schäffel F, Gorantla S, Bachmatiuk A and Schmidt O G 2012 Rolled-up nanomembranes as compact 3D architectures for field effect transistors and fluidic sensing applications Nano Lett. 13 213-8

[23] Yu X, Huang W, Li M, Comberiate T M, Gong S, Schutt-Aine J E and Li X 2015 Ultra-small, high-frequency, and substrate-immune microtube inductors transformed from 2D to 3D Sci. Rep. 59661
[24] Müller C, Bof Bufon C C, Navarro Fuentes M E, Makarov D, Mosca D H and Schmidt O G 2012 Towards compact threedimensional magnetoelectronics-magnetoresistance in rolled-up Co/Cu nanomembranes Appl. Phys. Lett. 100 022409

[25] Medina-Sánchez M, Ibarlucea B, Pérez N, Karnaushenko D D, Weiz S M, Baraban L, Cuniberti G and Schmidt O G 2016 High-performance three-dimensional tubular nanomembrane sensor for DNA detection Nano Lett. 16 4288-96

[26] Merces L, de Oliveira R F, de Camargo D H S and Bufon C C B 2017 Long-range coherent tunneling in physisorbed molecular ensembles J. Phys. Chem. C 121 16673-81

[27] Bof Bufon C C, Vervacke C, Thurmer D J, Fronk M, Salvan G, Lindner S, Knupfer M, Zahn D R and Schmidt O G 2014 Determination of the charge transport mechanisms in ultrathin copper phthalocyanine vertical heterojunctions J. Phys. Chem. C 118 7272-9

[28] Bof Bufon C C, Arias Espinoza J D, Thurmer D J, Bauer M, Deneke C, Zschieschang U, Klauk H and Schmidt O G 2011 Hybrid organic/inorganic molecular heterojunctions based on strained nanomembranes Nano Lett. $113727-33$

[29] Bendova M, Bof Bufon C C, Fomin V M, Gorantla S, Rümmeli M H and Schmidt O G 2016 Electrical properties of hybrid nanomembrane/nanoparticle heterojunctions: the role of inhomogeneous arrays J. Phys. Chem. C 120 6891-9

[30] Merces L, de Oliveira R F, Gomes H L and Bufon C C B 2017 The role of the electrode configuration on the electrical properties of small-molecule semiconductor thin-films $\mathrm{Org}$. Electron. 49 107-13

[31] Guo Q, Wang G, Chen D, Li G, Huang G, Zhang M, Wang X, Mei Y and Di Z 2017 Exceptional transport property in a rolled-up germanium tube Appl. Phys. Lett. 110112104

[32] de Oliveira R F, Merces L, Vello T P and Bufon C C B 2016 Water-gated phthalocyanine transistors: operation and transduction of the peptide-enzyme interaction $\mathrm{Org}$. Electron. 31 217-26

[33] Jalil A R et al 2016 Fully integrated organic nanocrystal diode as high performance room temperature $\mathrm{NO}_{2}$ sensor $A d v$. Mater. 28 2971-7

[34] de Oliveira R F, Merces L, Marques F, Teixeira-Neto E, de Camargo D H S and Bufon C C B 2018 Single-electron charging effects in hybrid organic/inorganic nanomembrane-based junctions J. Phys. Chem. C (https:// doi.org/10.1021/acs.jpcc.8b00233)

[35] Bof Bufon C C, Cojal González J D, Thurmer D J, Grimm D, Bauer M and Schmidt O G 2010 Self-assembled ultracompact energy storage elements based on hybrid nanomembranes Nano Lett. 10 2506-10

[36] Padma N, Maheshwari P, Bhattacharya D, Tokas R B, Sen S, Honda Y, Basu S, Pujari P K and Rao T C 2016 Investigations on substrate temperature-induced growth modes of organic semiconductors at dielectric/ semiconductor interface and their correlation with threshold voltage stability in organic field-effect transistors ACS Appl. Mater. Interfaces 8 3376-85

[37] Saleh A M, Hraibat S M, Kitaneh R M-L, Abu-Samreh M M and Musameh S M 2012 Dielectric response and electric properties of organic semiconducting phthalocyanine thin films J. Semicond. 33082002

[38] Hamam K J, Al-Amar M M, Mezei G, Guda R and Burns C A 2017 Dielectric response of branched copper phthalocyanine Mater. Res. Express 4095101

[39] Jarosz G, Signerski R and Brehmer L 2006 On dielectric spectra of thin copper phthalocyanine films Thin Solid Films $514287-91$ 
[40] Shi N and Ramprasad R 2007 Intrinsic dielectric properties of phthalocyanine crystals: an ab initio investigation Phys. Rev. B 75155429

[41] Shi N and Ramprasad R 2006 Dielectric properties of $\mathrm{Cu}-$ phthalocyanine systems from first principles Appl. Phys. Lett. 89102904

[42] Ramprasad R and Shi N 2006 Polarizability of phthalocyanine based molecular systems: a first-principles electronic structure study Appl. Phys. Lett. 88222903

[43] Groner M D, Elam J W, Fabreguette F H and George S M 2002 Electrical characterization of thin $\mathrm{Al}_{2} \mathrm{O}_{3}$ films grown by atomic layer deposition on silicon and various metal substrates Thin Solid Films 413 186-97

[44] George S M 2010 Atomic layer deposition: an overview Chem. Rev. 110 111-31

[45] Spahr H, Montzka S, Reinker J, Hirschberg F, Kowalsky W and Johannes H-H 2013 Conduction mechanisms in thin atomic layer deposited $\mathrm{Al}_{2} \mathrm{O}_{3}$ layers J. Appl. Phys. 114183714

[46] Sharma R, Bufon C C B, Grimm D, Sommer R, Wollatz A, Schadewald J, Thurmer D J, Siles P F, Bauer M and Schmidt O G 2014 Large-area rolled-up nanomembrane capacitor arrays for electrostatic energy storage $A d v$. Energy Mater. 41301631
[47] Hamam K J, Al-Amar M M, Mezei G, Guda R and Burns C 2014 High dielectric constant response of modified copper phthalocyanine J. Mol. Liq. 199 324-9

[48] Spahr H, Bülow T, Nowak C, Hirschberg F, Reinker J, Hamwi S, Johannes H-H and Kowalsky W 2013 Impact of morphological defects on the electrical breakdown of ultra thin atomic layer deposition processed $\mathrm{Al}_{2} \mathrm{O}_{3}$ layers Thin Solid Films 534 172-6

[49] Liu W, Zhou W, Liu S, Zhang C, Huang S, Li Y and San Hui K 2018 Electrical impedance performance of metal dry bioelectrode with different surface coatings Sensors Actuators A 269 515-23

[50] Peisert H, Schwieger T, Auerhammer J M, Knupfer M, Golden M S, Fink J, Bressler P R and Mast M 2001 Order on disorder: copper phthalocyanine thin films on technical substrates J. Appl. Phys. 90 466-9

[51] Iacovita C, Rastei M V, Heinrich B W, Brumme T, Kortus J, Limot L and Bucher J P 2008 Visualizing the spin of individual cobalt-phthalocyanine molecules Phys. Rev. Lett. 101116602

[52] Alessio P et al 2012 Molecular architecture and electrical properties in evaporated films of cobalt phthalocyanine J. Nanosci. Nanotechnol. 12 7010-20

[53] El-Nahass M M, El-Gohary Z and Soliman H S 2003 Structural and optical studies of thermally evaporated $\mathrm{CoPc}$ thin films Opt. Laser Technol. 35 523-31 